\title{
The Human Heart Electrophysiological Data Visualization: A Review
}

\author{
Fei Yang ${ }^{1}$, Kechao Wang ${ }^{2,3}$, Tiantian Wang ${ }^{2}$ and Lei Zhang*4 \\ ${ }^{1}$ School of Mechanical, Electrical and Information Engineering, Shandong University, Weihai, 264209, China \\ ${ }^{2}$ School of Computer Science and Technology, Harbin Institute of Technology, Harbin, 150001, China \\ ${ }^{3}$ School of Information Engineering, Harbin University, Harbin, 150086, China \\ ${ }^{4}$ School of Medicine, University of Pittsburgh, Pittsburgh, PA, 15212, USA
}

*Corresponding author: Lei Zhang, School of Medicine, University of Pittsburgh, Pittsburgh, USA.

\begin{abstract}
By the comprehensive mathematical description of cardiac biochemistry, bioelectricity and biophysics, cardiac computational models simulate function and behavior of the heart so that researchers can understand the underlying mechanism of cardiac activity. So, visualize the electrophysiological data is of great importance. In this paper, we review the progress for the development of the visualization method of the human heart electrophysiological data.
\end{abstract}

\section{Introduction}

Cardiac electrical activities are valuable for the investigation of complex heart diseases, e.g., arrhythmias, ischemia, and ventricular fibrillation, and can be invasively measured by medical devices like electrocardiogram (ECG). With the progress in programmed electrical modeling and simulation, electrophysiological simulation has gradually been a promising direction and an essential method for cardiac electrophysiology study to understand the mechanism and functionality of the heart. By simulating the cardiac electrical activities over a wide variety of scales from single ion channel proteins to whole organs, cardiac electrophysiological simulation can help in revealing the mechanisms of normal and abnormal cardiac electrical activities, interpreting of clinical data, and even designing drugs and therapeutic plans. Due to the complex structure of the heart, the large amount of data and the hidden electrophysiological feature, to observe the electrophysiological is a challenging task. Fortunately, researchers have been dedicated to propose visualization method to facilitate the research of electrophysiological simulation.

\section{Solution}

In this section, we present the solution of the electrophysiological data visualization. Hurmusiadis has developed a simulation system called Virtual Heart [1] for electrophysiological behavior demonstration. The proposed system was coded in C++ and OpenGL. Lu et al. simulate the wave propagation of human ventricular tissue with ischemia symptom through the increasing of concentration of extracellular $\mathrm{K}+$ and ischemic size. And they visually assess both 2D and 3D simulation results [2]. However, these systems did not take advantage of spatial information that can improving the demonstration of electrophysiological behavior. With the context information from the heart anatomical structure, one can easily understand the visualization results. Thus, to improve the 3D electrophysiological behavior visualization, we should consider both anatomical reference and electrophysiological measurements. In response to this question, Seemann [3] established heterogeneous three-dimensional anatomical and electro-physiological model of human atria. Wang et al. [4] have proposed a multi-dimensional transfer function to visualize cardiac electrophysiology simulation. In their work, an improved LH histogram method was proposed, and multi-dimensional transfer function design was discussed. With this method, boundary information was enhanced, and cardiac electrophysiology behavior was accurately demonstrated, as shown in Figure1. 


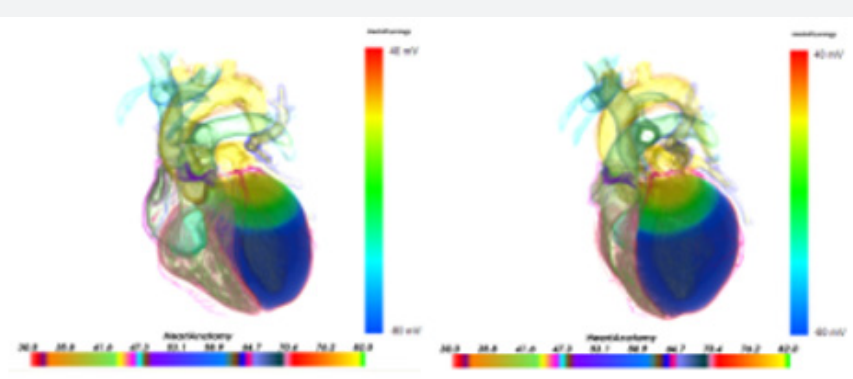

(a)

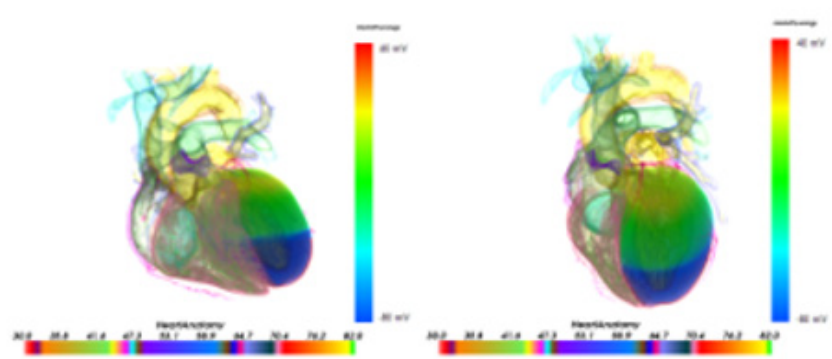

(b)

Figure 1: Rendering of electrophysiological simulation combined with anatomical model using multidimensional transfer function.

(a) excitation propagating in left ventricle at the time of $120 \mathrm{~ms}$.

(b) excitation propagating in left ventricle at the time of $250 \mathrm{~ms}$

Unfortunately, their implementation could not provide real-time rendering results, and results in poor user interaction performance. With our real-time rendering capability, their works could reflect the wave propagation and the time elapsing. To address this problem, Kharche et al. proposed a highperformance computing (HPC)+high-performance visualization (HPV) framework to study the 3D anatomically detailed model of clinical human atria electrophysiology [5]. However, the bottleneck of the communication of their method would drastically affect the visualization efficiency when the data transfer is frequent. In addition, the hardware and the software used in this work were not easily acquired. Thanks to the development of Graphics processing unit (GPU), Zhang et al. investigated image processing, optimization techniques for 4D (3D + time) cardiac image rendering [6]. They also proposed GPU-accelerated methodologies for multimodality 4-D medical image visualization and optical blending, along with real-time synchronization of dual-modality dynamic cardiac images. They also discussed transfer function design and data exploration for both dynamic volumetric magnetic resonance imaging (MRI) and ultrasound (US) cardiac data. Zhang et al. proposed a GPU-base ray-casting method and designed transfer function for visual evaluating the electrophysiological data [7]. They then proposed a multi-modality visualization method for electrophysiological data [8]. Their work was initially inspired by [4] and they used an improved Context-preserving model [9] to offer silhouette information for demonstrating the spatial relation of the tissue with the electrophysiological wave propagation and the anatomic structure of the human heart. Figure 2 shows an example of observing the electrophysiological simulation with anatomical reference of different setup. The proposed method was finally integrated into the G-Heart platform for GPU accelerated electrophysiological simulation and interactive multi-modality cardiac visualization [10]. Figure 3 demonstrates an example of electrophysiological data visualization in different time step (Figure 2\&3).
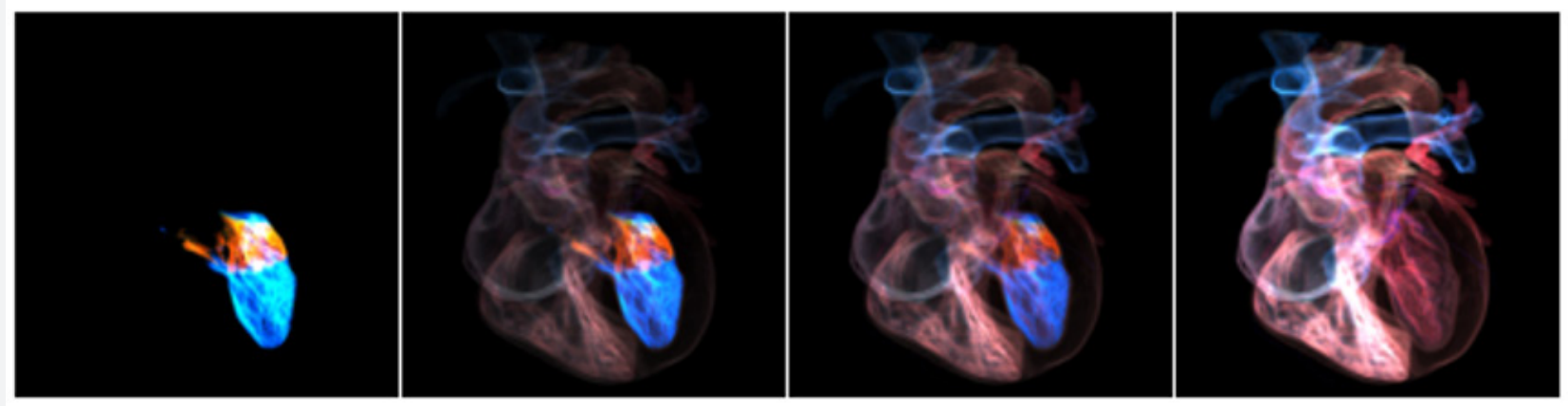

Figure 2: From left to right, different fusion setup of multi-modality visualization for electropysiological data [8]. 

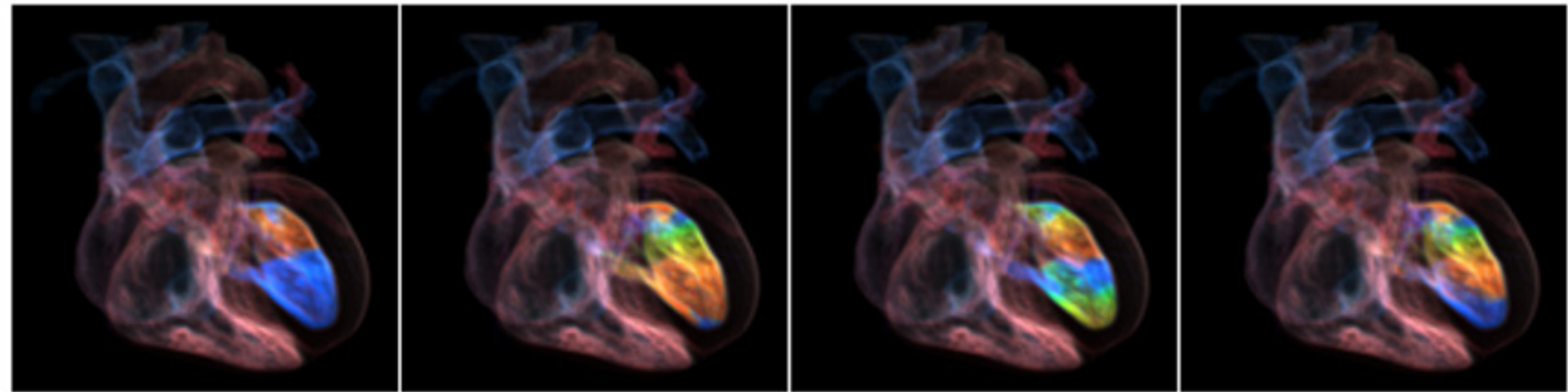

Figure 3: From left to right, visualization of electropysiological data in different time step [10].

In order to further improve the rendering speed, Yu et al. introduced an efficient GPU-based rendering algorithm for 4D electrophysiological data visualization [11]. The proposed rendering algorithm can greatly boost the visualization speed while keeping the high quality rendering results. To further uncover the organ level cardiac bioelectrical behavior, Yang et al. proposed a coupling ray radiation function (CRRF) for the dual pattern visualization of coupling of cardiac bioelectricity activity and static physical structure [12]. To study the dynamics of cardiac ischemic reentrant pathways, Heidenreich [13] proposed a 3D heterogeneous model for the regional ischemic human ventricles. The visualized simulation results predicted the appearance of figure-of-eight reentrant wave fronts that cross the central ischemic zone.

How can we boost the rendering speed? Are there new techniques to visually explore the electrophysiological data? These questions are still open questions for future investigation.

\section{Discussions and Perspectives}

We have introduced recent electrophysiological data visualization techniques. Volume rendering methods have been dominantly applied together with GPU techniques. In the foreseeable future, the electrophysiological data visualization will still be an active research area, since the massive amount of electrophysiological simulation data will be available and high rendering efficient and high-quality rendering method is in great need. With new techniques especially WebGL and cinematic rendering $[14,15]$ emerging, we can expect electrophysiological simulation data visualization methods of great rendering quality and rich user interaction to come.

\section{Conclusion}

Understanding the underlying mechanism of cardiac activities have benefited significantly from visualizing cardiac electrophysiological function data, which is expected to have an impact on diagnosis, therapy planning and prevention of cardiac disorders. Current visualized cardiac electrophysiological features have a remarkable biophysical detail. This review of the human heart electrophysiological data visualization has covered two major prospects: the rendering efficacy and the quality of visualization. The rendering efficacy will be further improved with the development of rendering hardware, e.g., GPU techniques. The quality of the visualization will be enhancing as well to meet the request of future electrophysiology research and cardiac healthcare.

\section{Acknowledgement}

Fei Yang was supported by the National Natural Science Foundation of China (NSFC) under Grant Nos. 61502275 and the Postdoctoral Science Foundation of China (No. 2017M622210).

\section{Conflicts of interest}

No conflict of interest.

\section{References}

1. Hurmusiadis V (2007) Virtual heart: Simulation-based cardiac physiology for education. In Computers in Cardiology: 65-68.

2. Lu WG, KQ Wang, WM Zuo, TJ Liu and HG Zhang (2009) Simulation of effects of ischemia in $3 \mathrm{~d}$ human ventricle. In Computers in Cardiology: 477-480.

3. Seemann G (2006) Heterogeneous three-dimensional anatomical and electro-physiological model of human atria. Philos Transact A Math Phys Eng Sci 364(1843): 1465-1481.

4. Wang Kuanquan, Fei Yang, Wangmeng Zuo, Na Ding and Henggui Zhang (2011) Effective transfer function for interactive visualization and multivariate volume data. In Biomedical Engineering and Informatics (BMEI) 1: 272-276

5. Kharche Sanjay, Gunnar Seemann, Lee Margetts, Joanna Leng, Arun $V$ Holden et al. (2008) Simulation of clinical electrophysiology in 3D human atria: a high-performance computing and high-performance visualization application. Concurrency and Computation: Practice and Experience 20(11): 1317-1328.

6. Zhang Q, Eagleson R, and Peters TM (2012) GPU-Based visualization and synchronization of 4-d cardiac MR and ultrasound images. IEEE Transactions on Information Technology in Biomedicine 16(5): 878890.

7. Zhang L, Gai C, Wang K, Lu W \& Zuo W (2011) GPU-Based highperformance wave propagation simulation of ischemia in anatomically detailed ventricle. Computing in Cardiology: 469-472.

8. Zhang L, Wang K, Zuo W, Wu Y and Han D (2012) GPU-based fusion method for 3D electrophysiological data visualization. In Computerized Healthcare (ICCH): 51-56

9. Wang K, Zhang L, Gai C and Zuo W (2011) Illustrative visualization of segmented human cardiac anatomy based on context-preserving model. In Computing in Cardiology 485-488.

10. Zhang L, Wang K, Zuo W and Gai C (2014) G-Heart: A GPU-based system for electrophysiological simulation and multi-modality cardiac visualization. Journal of Computers 9(2): 360-367.

11. Yu Shui, Shanzhuo Zhang, Kuanquan Wang, Yong Xia and Henggui Zhang (2017) An efficient and fast GPU-based algorithm for visualizing large volume of 4D data from virtual heart simulations. Biomedical Signal Processing and Control 35: 8-18.

12. Yang F, Zhang L, Lu W, Zuo W and Wang K (2017) Coupling Ray Radiation Function Based Biophysical Detailed Dual Pattern Cardiac Visualization. Journal of Medical Imaging and Health Informatics 7(5): 939-943. 
13. Heidenreich EA, Ferrero JM, Rodríguez JF (2012) Modeling the human heart under acute ischemia. In: Calvo Lopez B, Peña E, editors. PatientSpecific Comput Model 5: 81-103.

14. Dappa E, Higashigaito K, Fornaro J, Leschka S, Wildermuth S et al. (2016). Cinematic rendering-an alternative to volume rendering for 3D computed tomography imaging. Insights imaging 7(6): 849-856.
15. Berger F, Ebert LC, Kubik-Huch RA, Eid K, Thali MJ et al. (2018) Application of Cinematic Rendering in Clinical Routine CT Examination of Ankle Sprains. AJR Am J Roentgenol 211(4): 887-890. 